\title{
El retorno de la gripe: liderazgo, trabajo en equipo y anticipación
}

\section{Return of the flu: leadership, teamwork and foresight}

\author{
J. Rello ${ }^{\mathrm{a}, *}$ y J. Balcells ${ }^{\mathrm{b}}$ \\ a CIBERES y Servicio de Medicina Intensiva Hospital Vall d'Hebron, Clinical Research \& Innovation in Pneumonia and Sepsis (CRIPS) \\ - Vall d'Hebron Institut de Recerca, Universidad Autónoma de Barcelona, Barcelona, España \\ b Programa de ECMO Pediátrico y Neonatal, Servicio de Cuidados Intensivos Pediátricos, Hospital Vall d'Hebron, Barcelona, España
}

Recibido el 20 de mayo de 2011; aceptado el 24 de mayo de 2011

Liderazgo, trabajo en equipo y anticipación son tres valores característicos de la Medicina Intensiva. Aplicando estos valores, los intensivistas dieron una respuesta sin precedentes a la amenaza de la pandemia por influenza A H1N1 (2009)v, con implicaciones organizativas, mediáticas y científicas. Una muestra de ello es la generación de más de 20 publicaciones $^{1-24}$ que ponen de manifiesto los beneficios de iniciar precozmente oseltamivir para reducir la carga viral y abortar la respuesta inmunológica innata y adaptativa ${ }^{2,17}$ que genera daños colaterales en forma de lesión pulmonar aguda. Gracias a esta colaboración científica de investigadores del Grupo de Trabajo en Enfermedades Infecciosas de la Sociedad Española de Medicina Intensiva, Crítica y Unidades Coronarias (GTEI-SEMICYUC) sabemos que poblaciones como las embarazadas, los obesos, inmunodeprimidos o los asmáticos, además de adultos jóvenes y niños sanos, pueden evitar complicaciones con el uso apropiado e inmediato del oseltamivir ${ }^{19}$.

Sin posibilidad de una vacunación efectiva, embarazadas, pacientes jóvenes, a menudo sin comorbilidades, obesos, asmáticos o inmunodeprimidos ingresaron en 2009 con cuadros de hipoxemia refractaria con condensaciones pulmonares rápidamente progresivas (neumonías víricas primarias) o sin opacidades (broncoespasmo), con una intensidad que no habíamos conocido previamente en las $\mathrm{UCI}$ españolas. La mortalidad de estos pacientes en España

\footnotetext{
* Autor para correspondencia.

Correo electrónico: jrello@crips.es (J. Rello).
}

fue superior al $25 \%{ }^{19}$, aunque sin alcanzar la letalidad de Latinoamérica ${ }^{7}$.

El otoño de 2010 trajo una nueva ola de gripe, como se podía anticipar, y en diciembre las $\mathrm{UCl}$ volvieron a llenarse de pacientes con insuficiencia respiratoria grave y síndrome de dificultad respiratoria aguda (SDRA). En este número de Medicina Intensiva se publica una descripción de los primeros 300 casos del 2010-11 reportados al registro del $\mathrm{GTE}^{25}$. El estudio tiene importantes limitaciones, ya que no hay niños, no se hace un análisis sistemático de la presencia de otros virus respiratorios, no queda clara cuál es la distribución de casos por centros ni tampoco se compara con un grupo control (con los episodios reportados en los mismos centros en el 2009), por lo que resulta difícil establecer comparaciones y limita su interpretación o generalización.

Tres aspectos susceptibles de mejora llaman la atención: a) la importante representación de grupos de riesgo sin vacunación; $b$ ) la demora en el inicio de oseltamivir incluso en grupos de riesgo, particularmente en las embarazadas, y c) el uso injustificado de esteroides en pacientes con neumonía, a pesar de haberse demostrado ${ }^{16}$ que aumentan las sobreinfecciones y la mortalidad. Esto lleva a pensar que deberían organizarse campañas de difusión a la población general y a los profesionales sanitarios, incluyendo especialidades como Medicina de Familia o comadronas, con liderazgo de intensivistas que muestren nuestra experiencia en $\mathrm{UCl}$ y las contundentes evidencias científicas que permiten demostrar que estas muertes y costosos ingresos en cuidados intensivos son evitables.

Los primeros estudios post-pandemia de la temporada estacional $^{26}$ de 2010 confirman que la neumonía viral 
primaria por influenza A H1N1 (2009) ha vuelto a ser la forma de presentación más frecuente de la gripe en las $\mathrm{UCI}$, y todo indica que así será en los próximos años. A diferencia de 2009, se presentó más tarde, coexistiendo con algunos casos de influenza $B$, así como con frecuentes coinfecciones bacterianas o por otros virus. También se ha podido observar la presentación en forma de broncoespasmo/bronquiolitis grave con radiografía normal.

En el Hospital Vall d'Hebrón, el pico de gripe se desplazó de octubre a finales de diciembre y en niños, en 2010, se afectaron más los menores de dos años. La comparación de 52 ingresos en $\mathrm{UCl}$ de los dos años ${ }^{26}$ permite confirmar que el porcentaje de SDRA fue comparable, pero los porcentajes de pacientes con SOFA $>5$, APACHE II por encima de $10 \circ \mathrm{PaO}_{2} / \mathrm{FiO}_{2}<100$ fueron superiores en 2010, mostrando que los pacientes eran más graves. Las coinfecciones se multiplicaron por 4 en 2010 (alcanzando el 39,1\%), con el neumococo predominando en adultos y el virus respiratorio sincitial en casos pediátricos (42\%). A pesar del aumento de gravedad en la presentación, la mortalidad (31\% en intubados) no aumentó significativamente y la estancia media en $\mathrm{UCI}$ se redujo en cinco días. Es posible que ello sea debido a la experiencia acumulada en nuestro hospital, reflejada en la desaparición del uso de corticoides para la neumonía, la utilización de antivíricos de forma precoz y prolongada, la optimización de la ventilación incorporando la oxigenoterapia de alto flujo (Optiflow ${ }^{\circledR}$ ) en el manejo inicial ${ }^{27}$ y recurriendo a la ventilación de alta frecuencia oscilatoria (VAFO) en los casos más graves.

Las evidencias disponibles y la experiencia acumulada en estos dos años sugieren la necesidad de descartar de forma sistemática la presencia de virus gripales y otros virus respiratorios por técnicas de biología molecular en los pacientes que ingresan con insuficiencia respiratoria en $\mathrm{UCl}$, dada la posibilidad de utilizar antivíricos que disminuyen la carga viral y mejoran su pronóstico. Por otro lado, la oxigenoterapia convencional debería ser sustituida por dispositivos capaces de suministrar altos flujos de oxígeno (hasta $50 \mathrm{~L} / \mathrm{min}$ ) con un grado de calentamiento y humidificación óptimos $\left(\right.$ Optiflow $\left.{ }^{\circledR}\right)$. Por último, para evitar las muertes y los ingresos en $\mathrm{UCI}$ hay que incrementar la tasa de vacunación de los grupos de riesgo y utilizar el oseltamivir de forma precoz, como se hace en otras enfermedades infecciosas.

La inquietud por afrontar mejor una próxima pandemia y otras emergencias sanitarias debería empujarnos a la creación de una red de $\mathrm{UCl}$, tanto de adultos como de niños, siguiendo el ejemplo de las islas británicas, intercomunicadas en tiempo real con el fin de dar una respuesta inmediata a potenciales emergencias sanitarias.

\section{Conflicto de intereses}

El Dr. Rello ha sido coordinador del Registro Europeo de Casos Graves de Gripe A, financiado con 45.000 euros en OnMedic por la European Society of Intensive Care Medicine y ha representado a España por delegación del MSPSI en las teleconferencias de la OMS y el ECDC sobre gripe. El Dr. Rello es jefe del grupo 18 de CIBERES. El Dr. Rello ha sido asesor en gripe, de forma no remunerada, de la OMS, del MSPSI, y del Departament de Salut de la Generalitat de Cataluña.
Ni el Dr. Rello ni sus familiares han recibido ningún tipo de financiación específica para la gripe por parte de CIBERES, la industria farmacéutica, sociedades científicas, o la Administración Pública.

El Dr. Balcells ha representado a España, como intensivista pediátrico, en las teleconferencias de la OMS y ECDC. $\mathrm{Ni}$ el Dr. Balcells ni sus familiares han recibido ningún tipo de financiación específica para la gripe por parte de la industria farmacéutica, sociedades científicas, Administración Pública o CIBERES.

\section{Agradecimientos}

El Dr. Rello agradece la oportunidad de aprender y la colaboración continuada de los miembros del GTEI de la SEMICYUC, los esfuerzos y dedicación desinteresada de los profesionales de su equipo de investigación y la confianza de Ildefonso Hernández y su equipo de la Dirección General de Salud Pública del MSPSI.

\section{Bibliografía}

1. Rello J, Rodríguez A, Ibáñez P, Socias L, Cebrián J, Marqués A, et al. Intensive care adult patients with severe respiratory failure caused by influenza $A(\mathrm{H} 1 \mathrm{~N} 1) \mathrm{v}$ in Spain. Crit Care. 2009:R148.

2. Bermejo-Martín JF, Ortiz de Lejarazu R, Pumarola T, Rello J, Almansa R, Ramírez P, et al. The $\mathrm{H} 1$ and TH17 hypercytokinemia as early host response signature in severe pandemic influenza. Crit Care. 2009;13:R201.

3. Rello J, Pop-Vicas A. Clinical review: Primary influenza viral pneumonia. Crit Care. 2009;13:235-45.

4. Rodríguez A, Lisboa T, Díaz E, Rello J. De la seguridad a la prevención: gripe A $(\mathrm{H} 1 \mathrm{n} 1) \mathrm{v}$, un nuevo desafío para los intensivistas. Med Intensiva. 2009;33:265-6.

5. Boqué C, Rello J. Puntos clave para la orientación y manejo terapéutico de los casos graves de gripe A (H1N1)v en urgencias. Emergencias. 2009;21:370-5.

6. Falagas M, Vouloumanou EK, Baskouta E, Rafailidis I, Polyzos K, Rello J. Treatment options for 2009 H1N1 influenza: evaluation of the published evidence. Int $\mathrm{J}$ Antimicrob Chemother. 2010;35:421-30.

7. Rodríguez A, Socias L, Guerrero JE, Figueira JC, González N, Maraví-Poma E, et al. Gripe A pandémica en una unidad de cuidados intensivos: experiencia en España y Latinoamérica (Grupo Español de Trabajo de Gripe A Grave/Sociedad Española de Medicina Intensiva, Crítica y Unidades Coronarias). Med Intensiva. 2010;34:87-94.

8. Rodríguez A, Lisboa T, Rello J. GEGAG/SEMICYUC (Grupo Español de Trabajo de Gripe A Grave/SEMICYUC) Gripe A ( $\mathrm{H} 1 \mathrm{~N} 1) v$ pandémica en $\mathrm{UCl}$. ¿Qué hemos aprendido? Arch Bronconeumol. 2010;46:24-31.

9. Ariano R, Sitar D, Zelenitsky S, Zarychanski R, Pisipati A, Ahern $S$, et al. Enteric absorption and pharmacokinetics of oseltamivir in pandemic H1N1 influenza (2009) Associated Critical Illness. Can Med Assoc J. 2010;182:357-63.

10. Fowler RA, Webb SA, Rowan KM, Sprung CL, Thompson BT, Randolph $\mathrm{AH}$, et al. Early observational research and registries during the 2009-2010 influenza A pandemic. Crit Care Med. 2010;38:120-32.

11. Falagas M, Koletsi P, Vouloumanou E, Rafailidis R, Kapaskelis A, Rello J. Effectiveness and safety of neuraminidase inhibitors in reducing influenza complications: a meta-analysis of randomized controlled trials. J Antimicrob Chemother. 2010;65:1330-46. 
12. Díaz E, Rodríguez A, Martín-Loeches I, Lorente L, Martín MM, Pozo-Laderas J, et al. Impact of obesity in patients infected with 2009 influenza A (H1N1). Chest. 2011;139(2):382-6.

13. Martín-Loeches I, Papiol E, Rodríguez A, Zaragoza R, Granada A, et al. Acute kidney injury in critical ill patients affected by influenza A (H1N1) virus infection. Crit Care. 2011;15:R66.

14. Martín-Loeches I, Sánchez-Corral A, Díaz E, Granada R, Zaragoza R, Villavicencio C, et al. Community-Acquired Respiratory Coinfection in Critically III Patients With Pandemic 2009 Influenza A(H1N1). Virus Chest. 2010;139:555-62.

15. Martín-Loeches I, Rodríguez A, Bonastre J, Zaragoza R, Sierra R, Marqués $A$, et al. Severe pandemic $(H 1 N 1) v$ influenza $A$ infection: Report on the first deaths in Spain. Respirology. 2011;16(1):78-85.

16. Martín-Loeches I, Lisboa T, Rhodes A, Moreno RP, Silva E, Sprung $C$, et al. Use of early corticosteroid therapy on ICU admission in patients affected by severe pandemic (H1N1) $\mathrm{V}$ Influenza A infection. Intensive Care Med. 2011;37:272-83.

17. Bermejo-Martín JF, Martín-Loeches I, Rello J, Antón A, Almansa R, Xu L, et al. Host adaptive immunity deficiency (HAID) in severe pandemic influenza. Crit Care. 2010;14:R167.

18. InFACT: Global H1N1 Collaboration InFACT: a global critical care research response to H1N1 Lancet. 2010;375(9708):11-13.

19. Rodríguez A, Díaz E, Martín-Loeches I, Sandiumenge A, Candell A, Díaz JJ, et al. Impact of early oseltamivir treatment on outcome in critically ill patients with 2009 pandemic influenza A. JAC. 2011;66:1140-9.

20. Maraví-Poma E, Martín-Loeches I, Regidor E, Laplaza C, Cambra K, Aldunate S, et al. Severe 2009 A/H1N1v influenza in pregnant women in Spain. Crit Care Med. 2011;39: 945-51.

21. De Haro L, Ferrer R, Valles J. Neumonía y síndrome de distrés respiratorio del adulto por el virus influenza A (H1N1). Med Intensiva. 2009;33:455-8.

22. Chamorro C, Palencia E, Bodi MA, Garrido G. Valoración del potencial donante de órganos y tejidos en el seno de la pandemia por gripe A H1N1. Med Intensiva. 2010;34:127-33.

23. Riquelme $R$, Jiménez $P$, Videla $A$, López $H$, Chalmez J, Singanayagan A, et al. Predicting mortality in hospitalized patients with 2009 H1N1 influenza pneumonia. Int J Lung Tuberc Dis. 2011;15:542-6.

24. González-Vélez AE, Díaz-Agero-Pérez C, Robustillo-Rodela A, Cornejo-Gutiérrez AM, Pita-López MJ, Oliva-Iñiguez L, et al. Factores asociados a ingreso en $\mathrm{UCl}$ en pacientes hospitalizados por influenza pandémica A/H1N1 2009. Med Intensiva. 2011;35:463-9.

25. Rodríguez AH, Martín-Loeches I, Bonastre J, Olaechea P, Álvarez-Lerma F, Zaragoza R, et al. First Influenza season alter the 2009 pandemic influenza: Report of the 300 first cases in Spain. Med Intensiva. 2011;35:208-16.

26. Poulakou G, Souto J, Balcells J, Pérez M, Laborda C, Roca $O$, et al. First Influenza season after the 2009 pandemic influenza: Characteristics of ICU admissions in adults and children in Vall d'Hebron Hospital. Clin Microbiol Infect. 2011. Doi: $10.1111 / \mathrm{j} .1469-0691$.

27. Roca O, Riera J, Torres, Masclans JR. High flow oxygen therapy in acute respiratory failure. Respir Care. 2010;55: 408-13. 